\title{
Population X: Are the super-Eddington $X$-ray sources beamed jets in microblazars or intermediate mass black holes?
}

\author{
E. Körding, H. Falcke, and S. Markoff* \\ Max-Planck-Institut für Radioastronomie, Auf dem Hügel 69, 53121 Bonn, Germany \\ Received 27 August 2001 / Accepted 12 December 2001

\begin{abstract}
Recent X-ray observations reveal an increasing number of X-ray sources in nearby galaxies exceeding luminosities of $L_{\mathrm{x}} \gtrsim 2 \times 10^{39} \mathrm{erg} \mathrm{s}^{-1}$. Assuming isotropic emission, the Eddington limit suggests a population of intermediate-mass black holes of $M_{\bullet} \gg 10 M_{\odot}$. However, Markoff et al. (2001a) proposed that jets may be contributing to the X-ray emission from X-ray binaries (XRBs), implying that some X-ray sources may be relativistically beamed. This could reduce the required black hole masses to standard values. To test this hypothesis, we investigate a simple X-ray population synthesis model for X-ray point sources in galaxies with relativistic of X-ray point sources in nearby galaxies. We show that the current distributions are consistent with black hole masses $M_{\odot} \lesssim 10$ and bulk Lorentz factors for jets in microquasars of $\gamma_{\mathrm{j}} \sim 5$. Alternatively, intermediate mass black holes up to $1000 M_{\odot}$ are required which are distributed in a powerlaw with roughly $\frac{\mathrm{d} N}{\mathrm{~d} M} \sim M^{-2}$.
\end{abstract} \\ beaming and compare it with an isotropic emission model. The model is used to explain a combined data set
}

Key words. X-rays: binaries - accretion, accretion disks - black hole physics - radiation mechanisms: non-thermal

\section{Introduction}

In recent years $\mathrm{X}$-ray observations have revealed several off-nucleus ultra-luminous X-ray sources (ULXs) in the luminosity range $10^{39}-10^{40} \mathrm{erg} \mathrm{s}^{-1}$ within nearby galaxies (La Parola et al. 2001; Mizuno et al. 2001; Bauer et al. 2001; Colbert \& Mushotzky 1999). The Eddington limit for an accreting object with mass $M$ is $L_{\mathrm{Edd}} \approx$ $1.25 \times 10^{38} \frac{M}{M_{\odot}} \mathrm{erg} \mathrm{s}^{-1}$, which implies that these sources are super-Eddington for stellar mass objects. Some ULXs show spectral transitions from a soft spectrum to a hard power law and luminosity variability (e.g. Mizuno et al. 2001; Kubota et al. 2001), ruling out supernova remnants and supporting the idea that ULXs can be attributed to accreting black holes. To achieve the observed $\mathrm{X}$-ray luminosities with isotropically radiating accretion disks should require a population of intermediate-mass black holes of 50-500 $M_{\odot}$. As discussed in Kubota et al. (2001), however, the measured inner-disk temperatures of ULXs $\left(T_{\text {in }}=1.0-1.8 \mathrm{keV}\right)$ are too high for these masses and there is no established formation scenario for such intermediate-mass black holes.

The problems with isotropic emission models have already been discussed by King et al. (2001), where the authors propose some form of anisotropic emission as

Send offprint requests to: E. Körding,

e-mail: körding@mpifr-bonn.mpg.de

* Humboldt research fellow. an alternative. A beaming factor of ten already reduces the required mass of the black holes to expected values, but this is difficult to achieve with pure disk models. Recently Markoff et al. (2001a) suggested that the spectrum of some XRBs could be explained by a coupled disk/jet model, where some of the X-ray emission is produced by synchrotron and inverse-Compton radiation in a jet. This emission would naturally be relativistically beamed. Mirabel \& Rodríguez (1999) (see also Reynolds et al. 1997) have pointed out that a number of nearby galaxies should host microblazars - microquasars with relativistically beamed jets pointed towards the observer. We will here investigate whether such populations of microblazars or intermediate mass black holes can indeed explain current data on ULXs and constrain the basic parameters required for these models.

\section{Simple models}

\subsection{The jet/disk model}

Black hole candidate XRBs can exist in several states, the two most distinct of which are a high/soft state where the observed spectrum is soft and thermally-dominated, and a low/hard state dominated by a non-thermal hard power law spectrum (e.g. Nowak 1995). Which state an XRB is in seems to depend on the accretion rate. One scenario for the evolution of XRBs is that the inner part of the accretion disk consists of an optically thin, advection-dominated 
accretion flow (ADAF) existing up to a transition radius where the accretion flow turns into a standard (Shakura \& Sunyaev 1973) optically thick disk (Esin et al. 1997). The low/hard state seems to be accompanied by persistent radio jets with optically thick synchrotron emission extending up to the optical and near-infrared (Fender 2001). Synchrotron and inverse Compton emission from the jet could also be produced in the X-rays for low/hard and high/soft states (Markoff et al. 2001a; Markoff et al. 2001b).

For a self-consistent population synthesis model, we will have to take the jet and the disk separately into account. For simplicity we make some assumptions in order to calculate the relative importance of the two processes for the overall distribution of XRBs. These are:

- We only consider two main populations: neutron stars of mass $1.4 M_{\odot}$ and black holes within a mass range of $5-15 M_{\odot}$, where we fix the amount of active black holes $\left(>5 \times 10^{36} \mathrm{erg} \mathrm{s}^{-1}\right)$ to $13 \%$ of the number of active neutron stars (e.g. Tanaka \& Lewin 1995);

- While the spectral states are defined only for black holes, for simplicity we apply them also to neutron stars, and consider only the low/hard and soft/high states;

- The probability that a given XRB has the accretion rate $\dot{M}$ is given by $\mathcal{W}(\dot{M}) \sim \dot{M}^{\xi}$ which we assume as a power law with a cutoff representing the Eddington limit;

- The distribution of accretion rates and basic jet parameters are assumed to be identical for neutrons stars and black holes;

- Soft X-ray emission is produced by an isotropically radiating disk and a relativistically beamed jet as discussed below.

The crucial point for a simple population synthesis model including jet emission is how a specific accretion rate translates into an X-ray luminosity. Here we assume that the transition between the low and the high state happens at a critical accretion rate $\dot{M}_{\mathrm{C}}$ and the luminosities scale as follows: above $\dot{M}_{\mathrm{C}}$ the disk luminosity increases linearly with $\dot{M}$ as expected for a standard accretion disk. Below $\dot{M}_{\mathrm{C}}$, the disk luminosity increases with $\dot{M}^{2}$ as expected for optically thin ADAFs (Narayan \& Yi 1995; for a constant $\alpha$-parameter). Assuming that the jet power scales linearly with $\dot{M}$, the optically thick jet synchrotron emission will scale roughly as $L_{\mathrm{x}, \mathrm{jet}} \propto \dot{M}^{1.4}$ (Falcke \& Biermann 1995; Falcke \& Biermann 1999). For simplicity we assume that this scaling also holds for the optically thin flux. At high accretion rates this scaling must break down when a significant fraction of the jet power is radiated away. In this phase the radiated power can only increase linearly with jet power. In the jet model of Markoff et al. (2001a) and Markoff et al. (2001b) this happens in the high state, roughly at $\dot{M}>\dot{M}_{\mathrm{C}}$, where the jet is inverseCompton cooled (radiating soft X-rays) by the accretion disk. However, since models for the contribution of jets to the high state of XRBs are not yet very well developed, we simply fix the luminosity of the jet at $L_{\text {jet }}=\eta L_{\text {disk }}$ at $\dot{M}_{\mathrm{C}}$, where $\eta$ is a free parameter.

Hence, we use the following simple parameterization for the soft X-ray luminosity of accretion disk and jet:

$$
\begin{gathered}
L_{\mathrm{disk}}=\left\{\begin{array}{c}
\epsilon\left(\frac{\dot{M}}{\dot{M}_{\mathrm{C}}}\right) \dot{M} c^{2} \text { if } \dot{M}<\dot{M}_{\mathrm{C}} \\
\epsilon \dot{M} c^{2} \text { if } \dot{M}_{\mathrm{C}}<\dot{M}<\dot{M}_{\mathrm{Edd}}
\end{array}\right. \\
L_{\mathrm{jet}}=\left\{\begin{array}{ll}
\eta \epsilon\left(\frac{\dot{M}}{\dot{M}_{\mathrm{C}}}\right)^{0.4} \quad \dot{M}^{2} \text { if } \dot{M}<\dot{M}_{\mathrm{C}} \\
\eta \epsilon \dot{M} c^{2} \text { if } \quad \dot{M}_{\mathrm{C}}<\dot{M}<\dot{M}_{\mathrm{Edd}}
\end{array} .\right.
\end{gathered}
$$

In the following we set the radiative efficiency of the standard accretion disk to the canonical value of $\epsilon=0.1$. For a given mass $M$ the parameter $\dot{M}_{\text {Edd }}$ has been chosen such that the luminosity of the disk and the jet integrated over all angles is equal to $L_{\text {Edd }}$. For simplicity, the mass distribution of black holes is given by $\mathrm{d} N / \mathrm{d} M=\mathcal{V}(M)=$ const.

For a bulk Lorentz factor of the jet of $\gamma_{j}>1$ the jet emission depends on the angle to the line of sight as given by Lind \& Blandford (1985). If the emission in the rest frame of the jet follows a power-law with spectral index $\alpha$, the observed emission is proportional to $\delta^{2+\alpha}$, where the Doppler factor $\delta=\frac{1}{\gamma_{\mathrm{j}}(1-\beta \cos \Theta)}$. The probability of seeing an object with an emission exceeding $L$ when in the rest frame the jet emits $L_{\mathrm{loc}}$ is:

$P\left(L, L_{\mathrm{loc}}\right)=\frac{1-\beta}{\beta}\left(\left(\frac{L_{\max }}{L}\right)^{\frac{1}{2+\alpha}}-1\right)$

where $L_{\max }=\delta^{2+\alpha}(\Theta=0) L_{\text {loc }}$ is the maximum possible emission. To derive this we only consider the jet component pointing towards us and then integrate over all inclination angles. Since we only discuss jets with $\gamma_{\mathrm{j}}>2$, the emission of the counter-jet is largely negligible.

With this parameterization, the contribution of a single population of XRBs at a given accretion rate and mass has three parameters $\left(\gamma_{\mathrm{j}}, \dot{M}_{\mathrm{C}}, \eta\right)$, which are reasonably well constrained by the underlying models. Observations of microquasars show that the typical Lorentz factors are in the range $\gamma_{\mathrm{j}} \simeq 2-5$ (Mirabel \& Rodríguez 1999; Fender et al. 1999). The typical values for the critical accretion rate discussed in the literature are around $\dot{M}_{\mathrm{C}} \sim 0.1$ (Narayan \& Yi 1995) and we keep this parameter fixed. The jet efficiency can in principle be fairly high, but probably $\eta \lesssim 0.3$ (Falcke \& Biermann 1995, 1999).

At high accretion rates, the jets emits a factor $\eta$ less radiation in its rest frame compared to the disk, but beaming will lead to an amplification of the jet with respect to the disk for small inclination angles. Beaming is strongest for sources whose jets point within the beaming cone with half-opening angle of $1 / \gamma_{j}$ towards the observer. For example, already $\gamma_{\mathrm{j}}=3$ will beam the jet component in a fraction of $5.5 \%$ of the binaries by a factor of 20 . This is more than enough to make up for the less efficient emission mechanism. For $\gamma_{\mathrm{j}}=5$ a fraction of $2 \%$ binaries are beamed by a factor of 77 . Therefore, in the low-luminosity regime $\left(\ll \epsilon \eta \dot{M}_{\mathrm{C}}\right)$ jets should dominate (because of low radiative efficiency of ADAFs), and again dominate in the 
super-Eddington regime due to beaming. In the intermediate regime up to $L_{\mathrm{Edd}}$, the disk will be more prominent.

Now we can calculate a synthetic $\log N-\log L$ distribution for our model. The emission from the disk and the jet is described by Eq. (1). To reach a given luminosity $L$, only the difference $L-L_{\text {disk }}$ has to be reached by the jet due to boosting. The estimated number of sources with a luminosity greater than $L$ is given by:

$$
\begin{gathered}
N(L)=\sum_{i=N, B} \mathcal{N}_{i} \int \mathrm{d} M \int \mathrm{d} \dot{M} \mathcal{V}_{i}(M) \mathcal{W}_{i}(\dot{M}) . \\
P\left(L-L_{\text {disk }}(\dot{M}), L_{\text {jet }}(\dot{M})\right)
\end{gathered}
$$

where the sum goes over the two populations.

\subsection{The disk-only model}

Of course, with the model discussed above we can also investigate the alternative scenario, that the soft X-ray emission originates only from the accretion disk, by setting $\eta=0$. In this case we have to leave the upper end of the black hole mass distribution and its power law index as a free parameter to obtain the high luminosities observed, and we have $\mathrm{d} N / \mathrm{d} M=\mathcal{V}(M) \propto M^{\zeta}$.

With the isotropic disk emission the estimated number of sources with a luminosity greater than $L$ is given by:

$$
\begin{gathered}
N(L)=\sum_{i=N, B} \mathcal{N}_{i} \int \mathrm{d} M \int \mathrm{d} \dot{M} \mathcal{V}_{i}(M) \mathcal{W}_{i}(\dot{M}) . \\
\Theta\left(L-L_{\text {disk }}(\dot{M})\right)
\end{gathered}
$$

where $\Theta$ is the usual step-function.

\section{Data}

To put meaningful constraints on a beaming model it is essential to compare the low-luminosity (un-beamed) parent population with the high-luminosity (beamed) population. However, the published X-ray population of a single galaxy has only marginal statistics in the high-luminosity regime. To get a more general X-ray population in the low-luminosity regime up to $\approx 5 \times 10^{38} \mathrm{erg} / \mathrm{s}$, we combine data from the galaxies M 101, M 31 and M 82 (Pence et al. 2001; Di Stefano et al. 2001; Griffiths et al. 2000). These are three close $(D<10 \mathrm{Mpc})$ galaxies with good published Chandra data. To get better statistics at higher luminosities, we used the luminosity function compiled by Roberts \& Warwick (2000) from ROSAT data of 49 spiral galaxies from the XHFS-sample. The host galaxy types are spiral with the exception of the irregular M 82. However this does not seem to make a significant difference (see Figs. 1 and 2).

To avoid incompleteness near the detection limit, for each data set we only use X-ray sources with a luminosity of ten times the respective detection threshold. Chandra has a different bandpass (0.3-10 keV) than ROSAT (0.1$2.2 \mathrm{keV}$ ), so we extrapolated the ROSAT-luminosities to

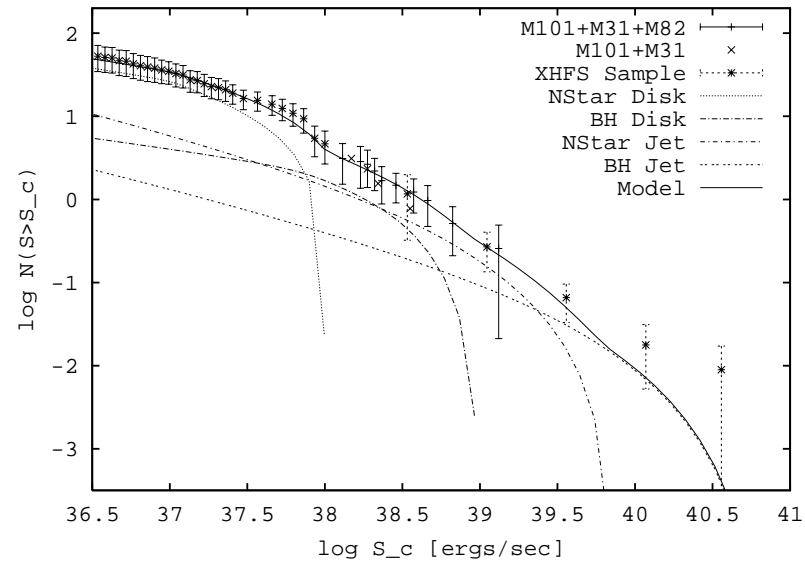

Fig. 1. Comparison of our model of the luminosity function with the data. The parameters are $\gamma_{\mathrm{j}}=5, \eta=0.3, \dot{M}_{\mathrm{C}}=0.1$, $\xi=1.4$. Also shown are the individual contributions of the disk and the jet for neutron stars and black holes.

the $2-8 \mathrm{keV}$ band. If the photon index is not fitted directly we used a common powerlaw with $\Gamma_{\mathrm{ph}}=1.7$. In some cases different values for $N_{\mathrm{H}}$ were used which we did not correct. According to Di Stefano et al. (2001), variations of $N_{\mathrm{H}}=0.6-1.5 \times 10^{21} / \mathrm{cm}^{2}$ and $\Gamma_{\mathrm{ph}}=1.2-2$ give differences in luminosity of about $20 \%$ which are not really significant in the $\log (N)-\log (S)$ plots shown here and should be statistically distributed (for ROSAT data the differences are higher).

As a reference galaxy we take M 101, to which we scale the populations of the other galaxies (i.e., the total number of sources in the overlapping luminosity bins). Combining these data sets assumes that the overall shape of the luminosity distribution is roughly universal. Clearly, the overall number of XRBs in each galaxy can depend strongly on the age of recent star formation, but the average slope of the luminosity function should be less sensitive to this. Since M 82 is irregular and has a much higher star forming rate than M 101 or M 31, we also show the data excluding M 82 , which is not significantly different.

To calculate errors we assume a standard deviation from the "general population" of $\sqrt{N}$ where $N$ is the number of detected sources, and use normal error propagation. Because we are showing a cumulative distribution, the errors for each point are not independent.

\section{Results}

To compare our simple model with the data we evaluate the integrals in Eqs. (2) and (3) numerically. The absolute normalization and the parameter $\xi$, which are entirely free, have been fit to the data at $L_{\mathrm{x}} \leq 10^{37} \mathrm{erg} \mathrm{s}^{-1}$. We obtain a best-fit value of the accretion rate index $\xi=1.4$ (note that the luminosity scales as $\dot{M}^{2}$ in this regime). As we only model neutron stars and black holes, the model fits could be affected at lower luminosities by other source populations like accreting white dwarfs and supernova remnants.

Figure 1 shows the result for our best-fit jet/disk model which requires $\gamma_{\mathrm{j}}=5$ and $\eta=0.3$, for the combined data 


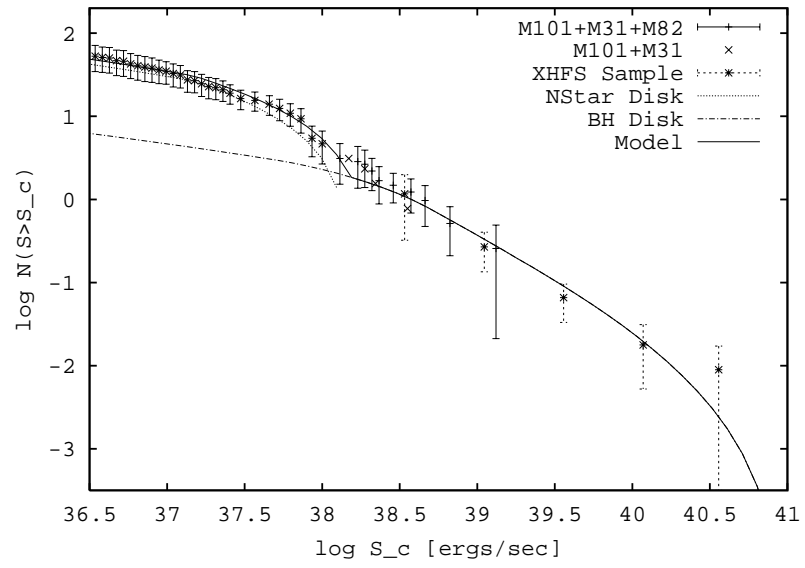

Fig. 2. Model with intermediate-mass black holes up to $1000 M_{\odot}$ and a mass distribution with power law index $\zeta=2$.

set discussed above. The Eddington limit for black holes (limited to $M<15 M_{\odot}$ ) and neutron stars shows up as breaks at the respective luminosities, and jets with $\gamma_{\mathrm{j}}=5$ are able to produce emission up to $10^{40} \mathrm{erg} \mathrm{s}^{-1}$ in significant numbers. The model is most sensitive to $\gamma_{\mathrm{j}}$ and $\eta$. Because the high luminosity domain depends linearly on $\eta$ while its dependence on $\gamma_{\mathrm{j}}$ goes as $\gamma_{\mathrm{j}}^{2.7}$, a slight decrease of $\gamma_{\mathrm{j}}$ can be compensated by an increase of $\eta$ and vice versa. For $\gamma_{\mathrm{j}}=5.8$ or $\gamma_{\mathrm{j}}=7.5$ we can find $\eta=0.2$ or $\eta=0.1$, but the fit gets progressively worse at higher Lorentz factors. Demanding $\eta \lesssim 0.3$ for the radiative efficiency of the jet sets a lower limit for $\gamma_{\mathrm{j}} \gtrsim 5$.

For the disk-only model, the sensitive parameters are the power law indices of the accretion rate and the mass distribution of the black holes. To fit the data, a mass distribution index of $\zeta \simeq 2$ is needed. The index of the accretion rate is the same as before (1.4), because the lower luminosities in the jet/disk model are also dominated by the disk. To explain the most luminous sources, the upper end of the black hole mass distribution must be extended at least up to $1000 M_{\odot}$. The results of the fit are shown in Fig. 2. For the lower luminosities the neutron stars dominate the luminosity function, while the black holes dominate at higher luminosities.

\section{Summary and discussion}

Using two very simple models for the evolution of XRBs, we calculate the luminosity distribution of X-ray point sources in nearby galaxies. We consider a jet/disk model based on Falcke \& Biermann (1999) and Markoff et al. (2001a), which can give rise to relativistically beamed emission from microblazars. Alternatively we also consider a purely isotropically radiating disk model.

Both models can in principle reproduce a combined luminosity function compiled from X-ray point source catalogs of three close galaxies and the XHFS spiral galaxy sample. However, as expected, the isotropic disk model requires a mass distribution of black holes extending out to $1000 M_{\odot}$ to explain the ULXs. On the other hand, a relativistic jet/disk model can fit the data with stellar mass black holes, if X-ray emitting jets with Lorentz factors $\gamma_{\mathrm{j}} \simeq 5$ are present in XRBs. In addition, a fraction of $\eta=10-30 \%$ of the total soft X-ray emission has to come from the jet rather than the accretion disk for an un-beamed XRB in the high state. This requires rather powerful jets but is not completely unreasonable. If only a fraction of the XRBs have relativistic jets, a slightly higher Lorentz factor or jet efficiency is needed. Boosting a 10 mJy Galactic XRB by a factor $\sim 10^{2}$ (for $\gamma \sim 5$ ) and placing it at $D \sim 3 \mathrm{Mpc}$ would yield only a faint $10 \mathrm{nJy}$ source and make radio detections difficult.

With the current statistics it is not possible to distinguish between the two different models, but it seems that microblazars provide at least a sensible alternative to the often discussed intermediate mass black hole scenario. Monitoring the spectral variability of the most luminous sources and further developing the XRB jet model should eventually help to disentangle the two scenarios.

Acknowledgements. The authors would like to thank R. Fender and an anonymous referee for useful suggestions.

\section{References}

Bauer, F. E., Brandt, W. N., Sambruna, R. M., et al. 2001, AJ, 122, 182

Colbert, E. J. M., \& Mushotzky, R. F. 1999, ApJ, 519, 89

Di Stefano, R., Kong, A., Garcia, M., et al. 2001 [astro-ph/0106254]

Esin, A. A., McClintock, J. E., \& Narayan, R. 1997, ApJ, 489, 865

Falcke, H., \& Biermann, P. L. 1995, A\&A, 293, 665

Falcke, H., \& Biermann, P. L. 1999, A\&A, 342, 49

Fender, R. P. 2001, MNRAS, 322, 31

Fender, R. P., Garrington, S. T., McKay, D. J., et al. 1999, MNRAS, 304, 865

Griffiths, R. E., Ptak, A., Feigelson, E. D., et al. 2000, Science, 290, 1325

King, A. R., Davies, M. B., Ward, M. J., Fabbiano, G., \& Elvis, M. 2001, ApJ, 552, L109

Kubota, A., Mizuno, T., Makishima, K., et al. 2001, ApJ, 547, L119

La Parola, V., Peres, G., Fabbiano, G., Kim, D. W., \& Bocchino, F. 2001, ApJ, 556, 47

Lind, K. R., \& Blandford, R. D. 1985, ApJ, 295, 358

Markoff, S., Falcke, H., \& Fender, R. 2001, A\&A, 372, L25

Markoff, S., Falcke, H., Fender, R., \& Biermann, P. 2001, in Proceeding of the 27th Cosmic Ray Conference (ICRC) (Hamburg, Germany), to appear

Mirabel, I. F., \& Rodríguez, L. F. 1999, ARA\&A, 37, 409

Mizuno, T., Kubota, A., \& Makishima, K. 2001, ApJ, 554, 1282

Narayan, R., \& Yi, I. 1995, ApJ, 452, 710

Nowak, M. A. 1995, PASP, 107, 1207

Pence, W. D., Snowden, S. L., Mukai, K., \& Kuntz, K. D. 2001, ApJ, 561, 189

Reynolds, C. S., Loan, A. J., Fabian, A. C., et al. 1997, MNRAS, 286, 349

Roberts, T. P., \& Warwick, R. S. 2000, MNRAS, 315, 98

Shakura, N. I., \& Sunyaev, R. A. 1973, A\&A, 24, 337

Tanaka, Y., \& Lewin, W. 1995, in X-ray Binaries (Cambridge University Press), Cambridge Astrophys. Ser., 26, 126 